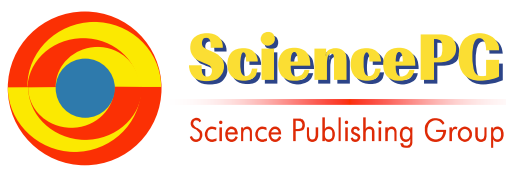

\section{Virtual antenna array theory and applications}

\author{
A. V. Ashikhmin ${ }^{1}$, Yu. G. Pasternak ${ }^{2}$, Yu. A. Rembovskiy ${ }^{1}$, S. M. Fedorov ${ }^{2}$ \\ ${ }^{1}$ JSC "IRCOS", Moscow, Russia \\ ${ }^{2}$ Faculty of radio engineering and electronics, Voronezh State Technical University, Voronezh, Russia
}

Email address:

info@ircos.ru (A. V. Ashikhmin), PasternakYG@mail.ru (Yu. G. Pasternak), zar36@yandex.ru (S. M. Fedorov)

\section{To cite this article:}

A. V. Ashikhmin, Yu. G. Pasternak, Yu. A. Rembovskiy, S. M. Fedorov. Virtual Antenna Array Theory and Applications. American Journal of Electromagnetics and Applications. Vol. 3, No. 1, 2015, pp. 1-11. doi: 10.11648/j.ajea.20150301.11

\begin{abstract}
In this article we would like to present a physical basis of virtual antenna array method which is based on electrodynamic principle of field source equivalence. Also, here are formulae and numerical relations for field description errors for the field at the edges of physical and virtual antenna arrays It is also demonstrated that the virtual antenna array method helps increase radio emitters angular coordinates accuracy even without data about antenna array carrier body geometry and material properties. Potential use of this method is also provided.
\end{abstract}

Keywords: Electromagnetic Fields, Diffraction, Approximation Methods, Antenna Array, Error Compensation

\section{Introduction}

Last years, different methods of virtual antenna array formation are coming into use or being developed. These methods enable to evaluate a spatial distribution of a field in a part of the space occupied by an actual antenna array (or real antenna array), i.e. the array which helps measure the field parameters near its components. Acc. to some publications pertaining to virtual antenna array formation methods, the use of this tool helps significantly increase resolution in terms of radio or acoustic system angular coordinates as well as to increase accuracy of emitters angular coordinates finding.

One of the first publications pertaining to narrow-band emitters direction finding by means of the virtual antenna arrays is publication [1]. There was an approach proposed where ROOT-MUSIC technique could be used, though this technique was originally applied only in linear equispaced antenna arrays and random configuration arrays. In this approach, the antenna system field of view is divided into sectors (especially it is interesting for $360 \mathrm{deg}$. sector), in which we search (using least squares method) transformation matrix of measured phase relations for the original and virtual antenna arrays. The matrix has some useful properties. In publication [1], there was a suggestion to change ROOT-MUSIC algorithm and use a virtual array signal subspace instead of spatial correlation matrix as well as selection of polynomial roots meeting the true signal acceptance angles. Further, the algorithm was successfully applied for correlated signals resolution.
To use Wiener interpolation techniques to form virtual antenna arrays was proposed in publication [2]. Let us set real and virtual array output signal vectors as $\mathbf{y}_{r}(t)$ and $\mathbf{y}_{v}(t)$, respectively (the arrays include $M$ and $M_{v}$ components respectively). The error of element output signal description for virtual antenna array depends on selection of calibration matrix $\mathbf{T}$ and its size $M_{v} \times M$ :

$$
\mathbf{e}(t)=\mathbf{y}_{v}(t)-\mathbf{T} \cdot \mathbf{y}_{r}(t)
$$

In publication [3] it was proposed to make interpolation between real and virtual arrays as a minimization problem with multiple constraints as inequations. This approach is based on minimization of sector interpolation error when there are a number of spatial filters in the other part of the space. It helps protect processing from the signals which arrive from sectors where no interpolation is performed.

In publication [4] there is one more interpolation procedure proposed: it is based on formation of a VAR (virtual antenna array) as a displaced copy of an actual AR (antenna array). In this case there is no need to select the number of VAR elements, distance between the elements and array orientation. Such displacement invariance is then used in ESPRIT technique. This method also requires sector-by-sector processing.

In publication [5] it was proposed to build the transformation matrix based on mitigation of signal arrival angle biasing. Subject to the members of the 2 nd order of decomposition of MUSIC output function into Taylor series, a 
formula to calculate direction finding error was established. This formula enabled to develop a conversion algorithm based on the least square method with a reduced error. Similar to Friedlander's approach, the signals outside of the transformation sector are ignored. Also, the author has expanded his approach to account for the final time of observation. Also, there was proposed a version of an algorithm to minimize root mean square deviation in direction finding.

In publication [6 and 7] there was an analysis of direction finding errors (biasing) which occur when transforming beams in space; a direction finding error expression was delivered. With this expression, a procedure was developed to mitigate evaluation dispersion caused by transformation of beams in space. Also it was the basis for how to select the number of virtual antenna elements. This criterion is based on analysis of array impulse IFFT and it is optimal in terms of virtual array aperture maximization with some limitations on the number of virtual elements. The criterion proposed by the author enables to achieve MSD close to Cramer-Rao bound.

The principle to form virtual antenna arrays or antenna multiplicity principle, as it was called by the authors, was proposed and studied in detail in publication [8]. The studies showed high effectiveness of direction finding antenna array calibration based on transition to equivalent linear equidistant antenna arrays. The proposed technique provides an accurate interpolation but still requires a dense grid of measured control vectors whose values shall be also measured very accurately. Reference generator field actual measurements (with auto-correlation matrix values distortion) describe noisy field and this will affect transformation quality The relation between the noise level and the number of modes for interpolation is established in publication [8]. Also there are some expressions which describe direction finding errors caused by the calibration noises and transformation. There was a formula to describe quality of MUSIC-like techniques used in multiplicity separation. This proposed formula used just those values which can be practically measured.

In publication [9] pertaining to Modal Space Processing (MSP) technique, it was demonstrated that: if we take Jacobi-Anger expansion $\exp \left(-i k_{0} x \cos (\theta)\right)$ to describe distribution of flat electromagnetic wave at the angle of $\theta$ to axis $x$ :

$$
\exp \left(-i k_{0} x \cos (\theta)\right)=\sum_{n=0}^{\infty} i^{n}(2 n+1) j_{n}\left(k_{0} x\right) P_{n}(\cos (\theta))
$$

where

$$
j_{n} \text { - Bessel spherical function }\left(j_{n}\left(k_{0} x\right) \approx \frac{\left(k_{0} x\right)^{n}}{1 \cdot 3 \cdot 5 \cdot \ldots \cdot(2 n+1)}\right.
$$

for $k_{0} x<<n$ )

$$
P_{n} \text { - Legendre function, }
$$

we can form a virtual antenna array. This expression describes distribution of a spatial electromagnetic wave along linear antenna array. It describes Fourier representation of $\exp \left(-i k_{0} x \cos (\theta)\right)$ in function basis $P_{n}(\cos (\theta))$ (mode) where $n=0,1, \ldots, \infty$. Parameters $\cos (\theta)$ and $k_{0} x$ are separated in this expression (they pertain to different functions) and therefore can be used for simplification of power spatial spectrum evaluation.

In publication [2] it was suggested to form virtual antenna arrays on the basis of original geometrical interpretation of statistical parameters of the second or higher orders (accumulants) of the signals received and processed by a real antenna array. In virtual array formation technique, the following formula is used for real control vector:

$$
\begin{aligned}
& a_{q, l}\left(\theta_{i}\right)=\exp \left\{j 2 \pi \left[\left(\sum_{j=1}^{l} x_{k_{j}}-\sum_{u=1}^{q-1} x_{k_{j+u}}\right) \cos \left(\theta_{i}\right) \cos \left(\phi_{i}\right)+\right.\right. \\
& +\left(\sum_{j=1}^{l} y_{k}-\sum_{u=1}^{q-1} y_{k_{j+u}}\right) \sin \left(\theta_{i}\right) \cos \left(\phi_{i}\right)+\left(\sum_{j=1}^{l} z_{k_{j}}-\sum_{u=1}^{q-1} z_{k_{j+u}}\right) . \\
& \left.\left.\cdot \sin \left(\phi_{i}\right)\right] / \lambda\right\}
\end{aligned}
$$

for emitter $i$ for virtual antenna array including $N^{q}$ elements with coordinates $\left(\sum_{j=1}^{l} x_{k_{j}}-\sum_{u=1}^{q-1} x_{k_{j+u}}\right)$, $\left(\sum_{j=1}^{l} y_{k_{j}}-\sum_{u=1}^{q-1} y_{k_{j+u}}\right),\left(\sum_{j=1}^{l} z_{k_{j}}-\sum_{u=1}^{q-1} z_{k_{j+u}}\right)$, where $1 \leq k_{j} \leq N$, $1 \leq j \leq q$ (with this the real antenna array includes $N$ elements).

In this publication it was demonstrated that formed virtual arrays can significantly (several times) increase radio system resolution in terms of angular coordinates and reduce side lobes level by tens of decibels.

In patent [10], a virtual antenna array uses two-dimensional (as per axis $x, y$ ) Lagrange's interpolation polynomials. A similar approach was used much earlier by authors of report [11]. The only difference there was that they used Kotelnikov's series.

Now let us specify some key features of the above VAR formation techniques.

1. Difractional distortion of the measured field caused by dispersion of waves on the antenna system and carrier body are accounted by means of calibration: i.e. to create a data base with control vectors $\mathbf{a}(\theta)$ for a finite set of emitter angular coordinates $\theta_{q}$ where $q=1, \ldots, Q$ as well as for a finite set of frequencies $f_{m}$, where $m=1, \ldots, M$. There are however some exceptions: techniques based on different types of interpolation of functions measured in different points.

2. Techniques where no calibration is used cannot increase accuracy of emitters angular coordinates finding.

3. Calibration is a labour-consuming and expensive procedure. In some cases it has no effect. Moreover, during equipment operation, re-calibration is often required.

4. The actual objective is to develop virtual antenna array formation techniques which require no calibration and specific knowledges in geometry and properties of dissipating objects like carrier's body, support mast, etc. 


\section{Problem Statement}

In some publications [12-15] there were studies how to approximate the field near 3D-diffuser after measuring its values by means of an antenna array in a finite number of closed loop points. With this, there was no need in geometrical data, material properties of the antenna array carrier body, underlying surface and other nearest diffusers.

As a result of those studies, there appeared several approaches and techniques to approximate the field near the diffuser, namely: techniques based on functions of complex variables (Cauchy integral, Laurent series, etc.); classic electrodynamic tools (Kirchhoff integral, auxiliary field emitters technique, highlights selection technique, etc.)

It was demonstrated that the use of approximated field spatial counts, which form a virtual antenna array, could be helpful to significantly increase radio system resolution in terms of angular coordinates, radio emitters angular coordinates finding accuracy and throughput.

Also it was demonstrated how to increase effectiveness of antenna array elements arrangement near the carrier body. This possibility was evaluated based on minimization of field phase distribution measurement error and azimuth coordinate of field phase distribution measurement error after field distortion caused by wave dissipation on the antenna system carriers body.

In this work we made an attempt to bring different ways of virtual antenna array formation into a system and substantiate them on the basis of electrodynamic principle of field source equivalents. It was shown that virtual antenna array theory could be based on Kirchhoff integration tools, Lorentz lemma and point emitters method.

Also, here are presented formulae and numerical relations for field description errors for the field at the edges of physical and virtual antenna arrays and on the loop where auxiliary field sources are located. Also, here it is demonstrated that description of a field at the edges of virtual antenna array based on Kirchhoff integration and description of a field as a superimposition of fields from several point (or linear) sources are equivalent.

It is also demonstrated that the virtual antenna array method helps increase radio emitters angular coordinates accuracy even without data about antenna array carrier body geometry and material properties. Potential application of this method in different radio systems is also described here

\section{Theory}

It is known [16] that function $u$ being a solution of reduced wave equation $\Delta u+k_{0}{ }^{2} u=0$ in internal points $P$ of space $V$ limited with closed Lyapunov-type surface $S$ can be defined in accordance with the third Green integral formula (Kirchhoff integral) using known values of function $u$ and its normal derivative $\partial u / \partial \vec{n}$ defined on surface $S$ :

$$
u(P)=\oint_{S}\left(G(P, Q) \frac{\partial u(Q)}{\partial \vec{n}}-u(Q) \frac{\partial G(P, Q)}{\partial \vec{n}}\right) d S_{Q},
$$

where

$$
\begin{aligned}
& k_{0} \text { - available space wave number } \\
& G(P, Q)=\frac{1}{4 \pi} \frac{\exp \left(-i k_{0} r_{P, Q}\right)}{r_{P, Q}} \text { - Green scalar function for a point }
\end{aligned}
$$
source

$r_{P, Q}$ - distance between observation point $P$ and current point $Q$ on surface $S$ (Green scalar function is differentiated in points $Q$ ).

Electromagnetic field in space $(V, S)$ with a vector projection $\vec{E}$ (or $\vec{H}$ ) being understood as $u$ may in a special case be a superimposition of incident wave fields $\left(\overrightarrow{E_{\text {inc }}}, \overrightarrow{H_{\text {inc }}}\right)$ and waves $\left(\overrightarrow{E_{\text {scatter }}}, \overrightarrow{H_{\text {scatter }}}\right)$ scattered by an object put inside the given space $(V, S)$.

Kirchhoff integral is a formal representation of principle of equivalent fields $(u(Q), \partial u(Q) / \partial \vec{n})$ known on closed surface $S$. It helps define the value of field $u(P)$ in any point inside space $(V, S)$ without any need for express data about the dissipating object (its geometry, material properties and position in the space $V$ ) as well as the data about external source, which create the incident wave field. Instead it is sufficient to know scalar field distribution $u$ and its normal derivative on the surface $S$.

Distribution of field $u(Q)$ and its normal derivative $\partial u(Q) / \partial \vec{n}$ on a closed surface $S$ are expressly related with distribution of the field defined on any internal closed surface $s$ (inside $V$ limited with external surface $S$ ) subject to definition of the normal vector to surface $S$.

The reverse problem - i.e. to define field $u(Q)$ (as well as its normal derivative $\partial u(Q) / \partial \vec{n})$ on closed surface $S$ with known values of field $u(P)$ on internal surface $s$ becomes much more difficult because it is related with solution of first kind integral-differential equation formed by means of Green third formula (Kirchhoff integral). If surfaces $S$ and $s$ do not intersect in any point (i.e. when $s$ is a strictly internal surface), then the values of Green scalar function and its normal derivative will be always finite and thus the analyzed equation will be a Fredholm equation.

In mobile direction finding equipment (Fig. 1), evaluation of radio emitter angular coordinates requires measurement of amplitudes $|u(P)|$ and phases $\arg (u(P))$ of (vertical, mostly)

projection of vector $\vec{E}$ of totalized electromagnetic field (formed by incident and scattered waves) in several points of closed loop $L$ (mostly, it is a circle, sometimes ellipse).

Values of field $u(Q)$ and its normal derivative $\partial u(Q) / \partial \vec{n}$ on integration surface $S$ and inside it are expressed through superimposition of fields of auxiliary point emitters $q$ set on external (to surface $S$ ) closed surface $\Gamma$, Fig. 1 . 
The radiation field of auxiliary point emitter in point $q \in \Gamma$, meets reduced wave equation (and thus meets the third Green integration formula) and is defined as follows:

$$
U(Q, q)=\frac{U_{q}}{4 \pi} \frac{\exp \left(-i k_{0} r_{Q, q}\right)}{r_{Q, q}},
$$

where

$U_{q}$ - point emitter complex amplitude

$r_{Q, q}$ - distance between point $Q$ and $q$.

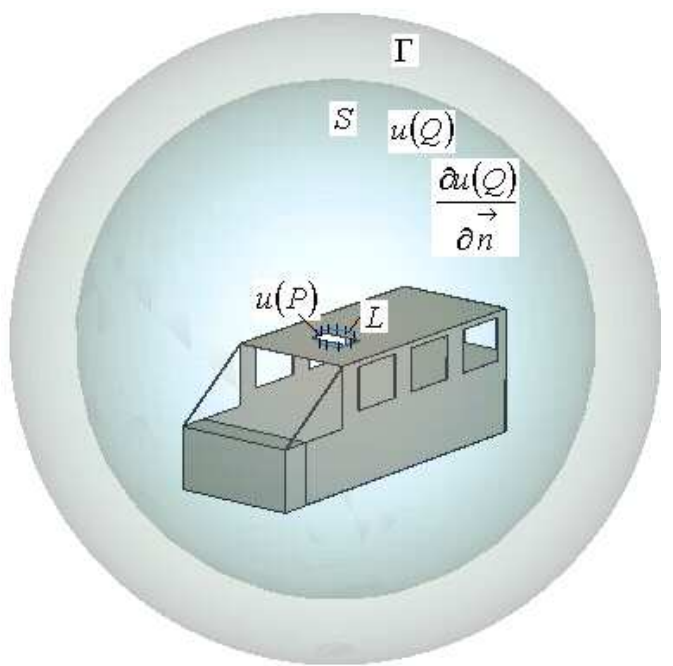

Figure 1. Arrangement of all scattering body elements (carrier's body and antenna array) inside integration surface $\mathrm{S}$ on which a virtual antenna array is formed. On external surface $\Gamma$ auxiliary point emitters are set.

When solving a practical problem related with extrapolation of a function describing a totalized field near a scatterer and when you know its values in the finite number of points, it is recommended to use such a finite number of auxiliary point emitters that is sufficient to achieve required field evaluation accuracy on surface $S$.

Now lets return to Lorentz lemma as a vector equivalent of the second Green formula [16:]

$$
\oint_{S}\left\{\vec{E}_{1}\left[\vec{H}_{2}, \vec{n}\right]-\vec{E}_{2}\left[\vec{H}_{1}, \vec{n}\right]\right\} d S=\int_{V}\left\{\left(\vec{E}_{2}, \vec{j}_{1}\right)-\left(\vec{E}_{1}, \vec{j}_{2}\right)\right\} d V,
$$

where

$\vec{E}_{1}, \vec{H}_{1}$ - electromagnetic field formed in $V$ by extrinsic currents $\overrightarrow{j_{1}}$;

$\vec{E}_{2}, \vec{H}_{2}$ - electromagnetic field formed in $V$ by extrinsic currents $\overrightarrow{j_{2}}$;

$S$ - limit of space $V$.

Note that, such expression for Lorentz lemma in the integral form involves no assumptions about properties of the media where the field is studied [16]. Thus, it may be both a non-homogeneous media and anisotropic media.

Lorentz lemma enables to express electromagnetic field inside volume $V$ through values of vector tangential components $\vec{E}$ and $\vec{H}$ on the surface of space $S$ [16].

Let's assume that $\left\{\overrightarrow{\mathrm{E}^{\alpha}}, \overrightarrow{\mathrm{H}^{\alpha}}\right\}$ is a field of electrical dipole $M_{0}$ with moment $\overrightarrow{e_{\alpha}}$ being a unit vector of Cartesian coordinates system $(\alpha=1,2,3)$. Now let us build Maxwell equations fundamental electrical matrices $\hat{\mathrm{E}}\left(M, M_{0}\right)$ and $\hat{\mathrm{H}}\left(M, M_{0}\right)$ whose lines are vector $\overrightarrow{\mathrm{E}^{\alpha}}$ and $\overrightarrow{\mathrm{H}}^{\alpha} \quad[16]$ :

$$
\hat{\mathrm{E}}=\| \begin{array}{|l}
\overrightarrow{\mathrm{E}}^{1} \\
\overrightarrow{\mathrm{E}}^{T} \\
\overrightarrow{\mathrm{E}}^{T} \\
\overrightarrow{\mathrm{E}}^{3}
\end{array}
$$

With Lorentz lemma we can express electric field vector in any random point of space $V$ being a vector equivalent of the third Green formula (Kirchhoff integral) [16]:

$$
\vec{E}\left(M_{0}\right)=\oint_{S}\left\{\begin{array}{l}
\hat{\mathrm{H}}\left(M, M_{0}\right)[\vec{E}(M), \vec{n}(M)]+ \\
+\hat{\mathrm{E}}\left(M, M_{0}\right)[\vec{H}(M), \vec{n}(M)]
\end{array}\right\} d S .
$$

The last expression has a very simple physical sense: the field in point $M_{0}$ inside a closed surface $S$, is formed by a total radiation of Huygens elements set on the given surface and associated tangent lines.

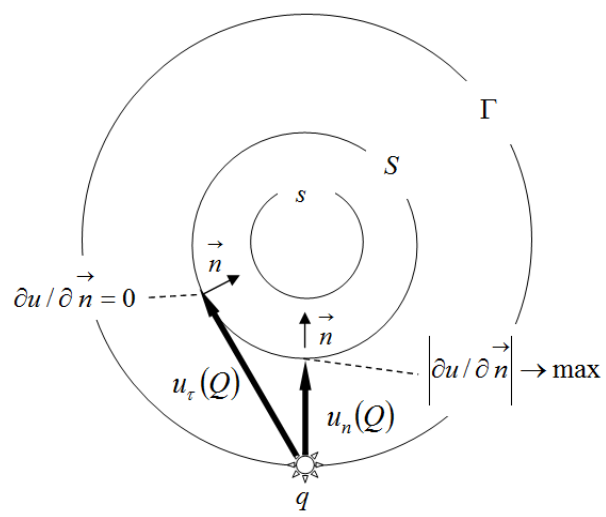

Figure 2. An illustration of decrease of normal derivative module of field $\partial \mathrm{u}(\mathrm{Q}) / \partial \overrightarrow{\mathrm{n}}$ at a deviation from anti-radial direction (Equivalence of covering of surface $S$ by Huygens elements)

When we create a virtual antenna array on surface $S$ (Fig. 1) by means of point emitters set on external surface $\Gamma$, we form a distribution of field $u(Q)$ and its normal derivative $\partial u(Q) / \partial \vec{n}$ on the first surface - and they will define field $u(P)$ in phase centers of the actual antenna system elements. When analyzing vector field $\vec{E}\left(M_{0}\right)$, each Huygens element 
set on surface $S$ will produce its maximum emission in the anti-radial direction (internal perpendicular line to the surface $S$ ). Similarly, when point emitters radiation direction deviates from the internal perpendicular line to the surface $S$, values of normal derivatives of function $\frac{\partial u(Q)}{\partial \vec{n}}$ and $\frac{\partial G(P, Q)}{\partial \vec{n}}$ will decrease in modulo when approaching to surface tangential line $S$, Fig. 2.

Therefore, when forming a virtual antenna array by means of auxiliary point emitters $q$ set on external surface $\Gamma$, surface $S$ becomes in fact covered by a system of infinitely small number of surface (scalar field) emitters $S$. Their radiation intensity is especially high towards internal perpendicular line to surface. This case is a full equivalent to the vector case with Huygens elements as elementary radiators.

Thus, the proposed way of virtual antenna array formation of surface $S$ by means of point emitters system $q$ set on external surface $\Gamma$ is physically adequate due to the above mentioned equivalency to the vector field meeting Lorentz lemma. The form selected to express the field of auxiliary emitter $U(Q, q)$ enables to adequately evaluate the field normal derivative on surface $S$.

In publication [17] there was demonstrated a system of functions $\varphi_{i}=\varphi\left(\vec{r}, \vec{R}_{i}\right)$ and $i=1,2, \ldots, N$ being a fundamental solution of reduced wave equation. This system met arrangement of auxiliary emitters $q$ with complex amplitudes ${ }_{U_{q_{i}}}$ in points with radius vectors $\vec{R}_{i}$ on auxiliary surface $\Gamma$. This surface entirely covers surface $s$ though does not have any common points with that. It is linearly independent and complete, and it can be used for an approximated solution of Helmholtz equation with given boundary conditions like

$$
u(\vec{r})=\sum_{i=1}^{N} U_{q_{i}} \varphi\left(\vec{r}, \overrightarrow{R_{i}}\right),
$$

where as functions $\varphi\left(\vec{r}, \vec{R}_{i}\right)$ Green scalar functions of point emitters $\vec{R}_{i}$ are used.

Note that the minimum problem associated with virtual antenna array is not just to find the value of field $u(Q)$ on closed surface $S$ covering the entire scatterer (Fig. 1) on its known values $u(P)$ in a finite number of points of closed loop $L$. The minimum problem here is to evaluate field values at least in the finite number of points on closed loop $\xi$ covering the points of antenna system elements phase centers (Fig. 3). Therefore let us review a more simple problem and review a plane on which three embedded loops are set: $\gamma$ auxiliary point emitters loop; $\boldsymbol{\xi}$ - Kirchhoff integration path, on which virtual antenna elements are set; and loop $L$ on which the actual antenna system is set.

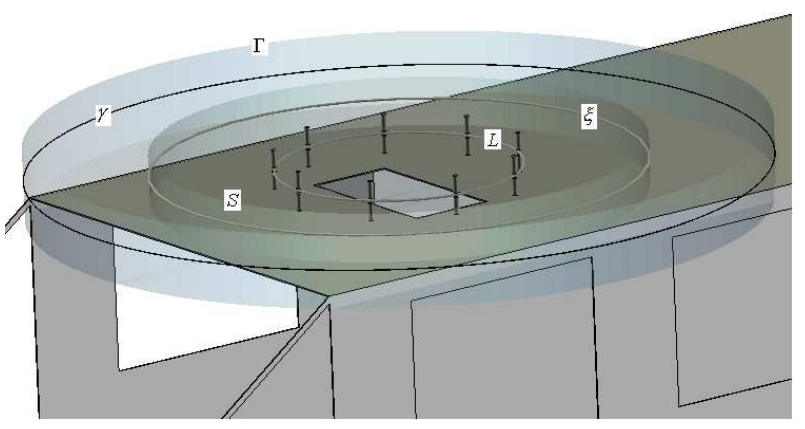

Figure 3. Shrinking surfaces $\Gamma$ and $S$ so that they could cover just the antenna array. Transition from cylinders with side surfaces $\Gamma$ and $S$ to loop $\gamma$ and $\xi$. On loop $L$ there are phase centers of antenna array elements

Such a shift is allowable because if the height of the cylinder with $S$ side surface gets reduced to zero, the impact onto Kirchhoff integral of the top and bottom cylinder bases will be compensated. This compensation will appear because derivatives $\frac{\partial u(Q)}{\partial \vec{u}}$ on the top and bottom cylinder base will be $\overrightarrow{\partial \vec{n}}$

opposite in sign due t countercurrent vectors $\vec{n}$ in relevant opposite points (the same is true for function $\frac{\partial G(P, Q)}{\partial \vec{n}}$ ). Values of function $u(Q)$ on the top and bottom cylinder bases in the opposite points will be the same (the same is true for function $G(P, Q)$ ). Therefore, when finding Kirchhoff integral, we can shift from surface integration $S$ to loop integration $\xi$.

It is worth mentioning, that in equivalent $2 \mathrm{D}$ problem (where loop $\gamma, \xi$ and $L$ are set in the same plane), some other emitters should be used. Instead of point emitter fields, it is better to use fields of infinitely long and infinitely thin (in the cross-section) linear emitters. This is recommended both for description of Green scalar function and auxiliary emitters fields (i.e. linear emitters, not point emitters as it was in the $3 \mathrm{D}$ problem).

$$
\begin{gathered}
G(P, Q)=-\frac{\pi i}{2} \cdot H_{0}^{1}\left(k_{0} r_{P, Q}\right), \\
U(Q, q)=-U_{q} \frac{\pi i}{2} \cdot H_{0}^{1}\left(k_{0} r_{Q, q}\right),
\end{gathered}
$$

where

$H_{0}^{1}(z)=J_{0}(z)+i N_{0}(z)$ - Hankel function of the first kind, zero order;

$J_{0}(z)$ and $N_{0}(z)$ - Bessel function and Neumann function respectively.

Thus, forming a virtual antenna array requires a solution of the following linear algebraic equations relative to unknown complex amplitudes $U_{q_{m}}$ of auxiliary linear emitters in points $q_{m} \in \gamma$ : 


$$
\sum_{m=1}^{N} U_{q_{m}} \cdot \sum_{i=1}^{I}\left(\begin{array}{l}
G\left(P_{n}, Q_{i}\right) \cdot \frac{\partial \varphi_{m}\left(Q_{i}, q_{m}\right)}{\partial \vec{n}}- \\
-\varphi_{m}\left(Q_{i}, q_{m}\right) \cdot \frac{\partial G\left(P_{n}, Q_{i}\right)}{\partial \vec{n}}
\end{array}\right) \Delta \xi_{i}=u\left(P_{n}\right), n=1,2, . . ., N
$$

where

$G\left(P_{n}, Q_{i}\right)$ - Green scalar function for linear emitter in point $Q_{i} \in \xi$ creating a field in point $P_{n} \in L$ of the phase center of the $n$-th element of the actual antenna array.

$I$ - the number of loop $\xi$ splittings when finding Kirchhoff integral.

$\varphi_{m}\left(Q_{i}, q_{m}\right)$ - value of the $m$-th basic function (Green scalar function for free space with an emitter in point $q_{m}$ ) in point $Q_{i}$ of loop $\xi$.

$\Delta \xi_{i}$ - length of circle element $\xi$.

$u\left(P_{n}\right)$ - the field measured by means of the $n$-th element of the actual antenna array in point $P_{n}$.

From the given system of algebraic linear equations, it can be seen that the fields on closed loop $L, \xi$ and $\gamma$ are rigidly bound between each other. Thus, we can assume that virtual antenna array on loop $L$ can be formed much easierthrough mere approximation of field with a linear combination of fields from auxiliary linear emitters (or, when simplified, point emitters) $q$ set on external loop $\gamma$, provided that the result of their superimposition in a number of observation points on internal loop $L$ is known (i.e. field $u(P)$ was measured by means of the actual antenna system).

The field on loop $\xi$ can be also described through complex variable analytical functions $z$ because if we know some values of the analytical function on closed loop $L$, we can restore its values in the entire complex plane.

We have found an effective way to form virtual antenna arrays - to use an analytical continuation of the field measured with antenna system by means of complex variable analytical functions $z=x+i y$, namely by means of Cauchy integral or Laurent series. This approach is applicable only in case of rather small electrical sizes of the space where the virtual array is formed.

On loop of equi-distant circular antenna array with radius $R$ a $E_{z}$-component can be described on the basis of measured field values $U_{1}, U_{2}, U_{3}, \ldots, U_{N}$, with use of following polynomial:

$$
U_{A A}(z=R \cdot \exp [i \varphi])=\sum_{n=1}^{N+1} B_{n-1} \cdot \exp [i(n-1) \varphi /(N+1)]
$$

which coefficients are found by the least squares method.

Using Poisson integral (specific case of Cauchy integral for a circumferential loop), we can compile an integral equation of the 1st kind to link the fields of the actual and virtual antenna arrays:

$$
\begin{aligned}
& U_{A P}(z=R \cdot \exp [i \varphi])=\frac{1}{2 \pi} \int_{0}^{2 \cdot \pi} U_{V A ?}(r \cdot \exp [i \psi]) \times \\
& \times \frac{r^{2}-R^{2}}{r^{2}-2 r R \cdot \cos (\psi-\varphi)+R^{2}} d \psi= \\
& =\frac{1}{2 \pi} \int_{0}^{2 \cdot \pi}\left\{\sum_{k=1}^{K+1} X_{k-1} \cdot \exp [i(k-1) \cdot \psi /(K+1)]\right\} \times \\
& \times \frac{r^{2}-R^{2}}{r^{2}-2 r R \cdot \cos (\psi-\varphi)+R^{2}} d \psi
\end{aligned}
$$

This equation can be solved by means of collocation after Tikhonov regularizations (or limited damage technique).

The other way of field approximation is based on expansion of function $U_{A A}(z)$ in loop $R-\delta \leq r \leq R+\delta$ (where $\delta<<R$ ) into Laurent series.

$$
U_{A A}(z)=\sum_{k=-\infty}^{\infty} c_{k} z^{k}, \text { where } c_{k}=\frac{1}{2 \pi i} \int_{\Gamma} \frac{U(\zeta) d \zeta}{\zeta^{k+1}} .
$$

Then, we apply (3) and find derivatives of function $U_{A A}(z)$ in radial directions of $\partial U(\varphi, r) / \partial r, \partial^{2} U(\varphi, r) / \partial r^{2}$ etc., and find the value of function $U_{V A R}(\varphi, R+L)$ by means of Taylor series section:

$$
\begin{aligned}
& U(\varphi, R+L) \approx U(\varphi, R)+L \cdot \partial U(\varphi, r) / \partial r+ \\
& +0.5 L^{2} \cdot \partial^{2} U(\varphi, r) / \partial r^{2}+\ldots
\end{aligned}
$$

Considering that the fields on flat loop $L, \xi$ and $\gamma$ are related with one another in the following ratio:

$$
\begin{aligned}
& u(P)=\oint_{\xi}\left(H_{0}^{1}\left(k_{0} r_{P, Q}\right) \frac{\partial U_{\Sigma}(Q)}{\partial \vec{n}}-U_{\Sigma}(Q) \frac{\partial H_{0}^{1}\left(k_{0} r_{P, Q}\right)}{\partial \vec{n}}\right) d \xi_{Q}= \\
& =\oint_{\gamma} U_{q}\left(x_{q}, y_{q}, z_{q}\right) H_{0}^{1}\left(k_{0} r_{P, q}\right) d \gamma_{q},
\end{aligned}
$$

and represent periodical functions of azimuth angle, then the following Fourier series will be respectively related:

$$
\begin{aligned}
& u(P)=\sum_{n=-\infty}^{\infty} a_{n} \exp \left(\operatorname{in} \varphi_{L}\right)=\oint_{\gamma} \sum_{n=-\infty}^{\infty} c_{n} \exp \left(\operatorname{in} \varphi_{\gamma}\right) H_{0}^{1}\left(k_{0} r_{P, q}\right) d \gamma_{q}= \\
& =\sum_{n=-\infty}^{\infty} c_{n} \oint_{\gamma} \exp \left(\operatorname{in} \varphi_{\gamma}\right) H_{0}^{1}\left(k_{0} r_{P, q}\right) d \gamma_{q},
\end{aligned}
$$

where

$$
\begin{aligned}
& a_{n}=\frac{1}{2 \pi} \int_{-\pi}^{\pi} u(P) \exp \left(-i n \varphi_{L}\right) d \varphi_{L}, \\
& c_{n}=\frac{1}{2 \pi} \int_{-\pi}^{\pi} U_{q}(q) \exp \left(-\operatorname{in} \varphi_{\gamma}\right) d \varphi_{\gamma},
\end{aligned}
$$

$\varphi_{L}$ and $\varphi_{\gamma}$ are azimuth coordinates of point $P \in L$ and $q \in \gamma$ respectively. 
Since there is a fast oscillating (with $n \rightarrow \infty$ ) function $\exp \left(\operatorname{in} \varphi_{\gamma}\right)$

under integral $\oint_{\gamma} \exp \left(\right.$ in $\left.\varphi_{\gamma}\right) H_{0}^{1}\left(k_{0} r_{P, q}\right) d \gamma_{q}$, whose modulus will quickly fall as far as $|n|$ will grow, then to describe function $u(P)$ by means of factors $c_{n}$ (with the given accuracy level) will require much less members of the series than it would take for approximation of $U_{q}(q)$.

Let us use some factors $b_{n}=\frac{1}{2 \pi} \int_{-\pi}^{\pi} U_{Q}(Q) \exp \left(-i n \varphi_{\xi}\right) d \varphi_{\xi}$ to expand function describing field distribution $U_{Q}$ on loop $\xi$ into Fourier series. Then it would be practical to use the following ratio to link the fields on loop $\gamma$ and $\xi$ :

$$
\begin{aligned}
& U_{Q}(Q)=\sum_{n=-\infty}^{\infty} b_{n} \exp \left(\operatorname{in} \varphi_{\xi}\right)=\oint_{\gamma} \sum_{n=-\infty}^{\infty} c_{n} \exp \left(\operatorname{in} \varphi_{\gamma}\right) H_{0}^{1}\left(k_{0} r_{Q, q}\right) d \gamma_{q}= \\
& =\sum_{n=-\infty}^{\infty} c_{n} \oint_{\gamma} \exp \left(\operatorname{in} \varphi_{\gamma}\right) H_{0}^{1}\left(k_{0} r_{Q, q}\right) d \gamma_{q} .
\end{aligned}
$$

Fourier series factors $a_{n}, b_{n}$ and $c_{n}$ describe functions on loop $L, \xi$ and $\gamma$ that meet Helmholtz equation and, thus, can be continuously differentiated at least twice. Therefore due to conclusion from Riemann-Lebesgue theorem [10] the following equations will be true:

$$
\left|a_{n}\right|<\frac{C_{1}}{n^{2}}, \quad\left|b_{n}\right|<\frac{C_{2}}{n^{2}}, \quad\left|c_{n}\right|<\frac{C_{3}}{n^{2}},
$$

where $C_{1}, C_{2}$ and $C_{3}$ are constant values.

A specific feature of dependency $I_{n}(n)$ and $J_{n}(n)$ is that they can be rather accurately approximated with functions like $\alpha \cdot \exp (-\beta \cdot n)$. In a specific case with $f=25 \mathrm{MHz}$, $r_{L}=0.5 \mathrm{~m}, r_{\gamma}=5 \mathrm{~m}$ for description of field on loop $L$, the $I_{n}(n)$ will be very close to $\alpha_{1} \cdot \exp \left(-\beta_{1} \cdot n\right)$ with $\alpha_{1}=3.5$ and $\beta_{1}=2.55$ at $n \in[3 ; 13]$. In a specific case with $\alpha_{2}=1.9$, and $\beta_{2}=1.4$ for description of field on loop $\xi$ with $r_{\xi}=1.5 \mathrm{~m}$ and value of $r_{\gamma}=5 \mathrm{~m}$, value $J_{n}(n)$ are well approximated by function $\alpha_{2} \cdot \exp \left(-\beta_{2} \cdot n\right)$ on frequency $f=25 \mathrm{MHz}$ at $n \in[3 ; 13]$.

Therefore, when approximizing the fields on loop $L, \xi$ and $\gamma$ via a finite number of Fourier harmonics, the following limiting functions can be used:

$$
\begin{gathered}
a_{n} \exp \left(\operatorname{in} \varphi_{L}\right)<\frac{C_{1}}{n^{2}}, \\
b_{n} \exp \left(\operatorname{in} \varphi_{\xi}\right)<\frac{C_{2}}{n^{2}}, \\
c_{n}<\frac{C_{3}}{n^{2}},
\end{gathered}
$$

$$
\begin{aligned}
& \oint_{\gamma} \exp \left(\operatorname{in} \varphi_{\gamma}\right) H_{0}^{1}\left(k_{0} r_{P, q}\right) d \gamma_{q}<\alpha_{1} \cdot \exp \left(-\beta_{1} \cdot n\right), \\
& \oint_{\gamma} \exp \left(\operatorname{in} \varphi_{\gamma}\right) H_{0}^{1}\left(k_{0} r_{Q, q}\right) d \gamma_{q}<\alpha_{2} \cdot \exp \left(-\beta_{2} \cdot n\right) .
\end{aligned}
$$

Now let us find the maximum error of evaluation of totalized $\sum_{n=-\infty}^{\infty} c_{n} \oint \exp \left(\operatorname{in} \varphi_{\gamma}\right) H_{0}^{1}\left(k_{0} r_{P, q}\right) d \gamma_{q}$ provided that the number of $n \in[-N ; N]$ harmonics is finite:

$$
\begin{aligned}
& \delta u_{c}<2 C_{3} \alpha_{1} \sum_{n=N+1}^{\infty}\left[\frac{\exp \left(-\beta_{1} n\right)}{n^{2}}\right]= \\
& =2 C_{3} \alpha_{1}\left\{\sum_{n=1}^{\infty}\left[\frac{\exp \left(-\beta_{1} n\right)}{n^{2}}\right]-\sum_{n=1}^{N}\left[\frac{\exp \left(-\beta_{1} n\right)}{n^{2}}\right]\right\}= \\
& =2 C_{3} \alpha_{1}\left\{-\int_{0}^{\exp \left(-\beta_{1}\right)} \frac{\ln (1-t)}{t} d t-\sum_{n=1}^{N}\left[\frac{\exp \left(-\beta_{1} n\right)}{n^{2}}\right]\right\}= \\
& =2 C_{3} \alpha_{1}\left\{L i_{2}\left(\exp \left(-\beta_{1}\right)\right)-\sum_{n=1}^{N}\left[\frac{\exp \left(-\beta_{1} n\right)}{n^{2}}\right]\right\}
\end{aligned}
$$

where $\quad L i_{2}(x)=\sum_{n=1}^{\infty}\left[\frac{x^{n}}{n^{2}}\right]=-\int_{0}^{x} \frac{\ln (1-t)}{t} d t \quad$ is $\quad$ a $\quad 2$ nd $\quad$ order polylogarithm from argument $x$, whereas $|x|<1$.

Now let us find the maximum error of evaluation of totalized $\sum_{n}^{\infty} a_{n} \exp \left(i n \varphi_{L}\right)$ provided that the number of $\sum_{n=-\infty}^{\infty} a_{n} \exp \left(\operatorname{in} \varphi_{L}\right)$ harmonics is finite: $n \in[-N ; N]$ :

$$
\begin{aligned}
& \delta u_{a}<2 C_{1} \sum_{n=N+1}^{\infty}\left[\frac{1}{n^{2}}\right]=2 C_{1}\left\{\sum_{n=1}^{\infty}\left[\frac{1}{n^{2}}\right]-\sum_{n=1}^{N}\left[\frac{1}{n^{2}}\right]\right\}= \\
& =2 C_{1}\left\{\frac{\pi^{2}}{6}-\sum_{n=1}^{N}\left[\frac{1}{n^{2}}\right]\right\}
\end{aligned}
$$

The maximum error of evaluation of totalized $\sum_{n=-\infty}^{\infty} c_{n} \oint_{\gamma} \exp \left(\operatorname{in} \varphi_{\gamma}\right) H_{0}^{1}\left(k_{0} r_{Q, q}\right) d \gamma_{q} \quad$ with a finite number of harmonics $n \in[-N ; N]$ will be:

$$
\delta U_{c}<2 C_{3} \alpha_{2}\left\{-\int_{0}^{\exp \left(-\beta_{2}\right)} \frac{\ln (1-t)}{t} d t-\sum_{n=1}^{N}\left[\frac{\exp \left(-\beta_{2} n\right)}{n^{2}}\right]\right\} .
$$

Thus, when $N$ increases from 3 to 9 , the field description error for loop $L$ (at $25 \mathrm{MHz}$ frequency) decreases more than by 7 orders; the field description order for loop $\xi$ decreases more than by 4 orders and this confirms that the auxiliary field emitters technique is very effective in field description. 


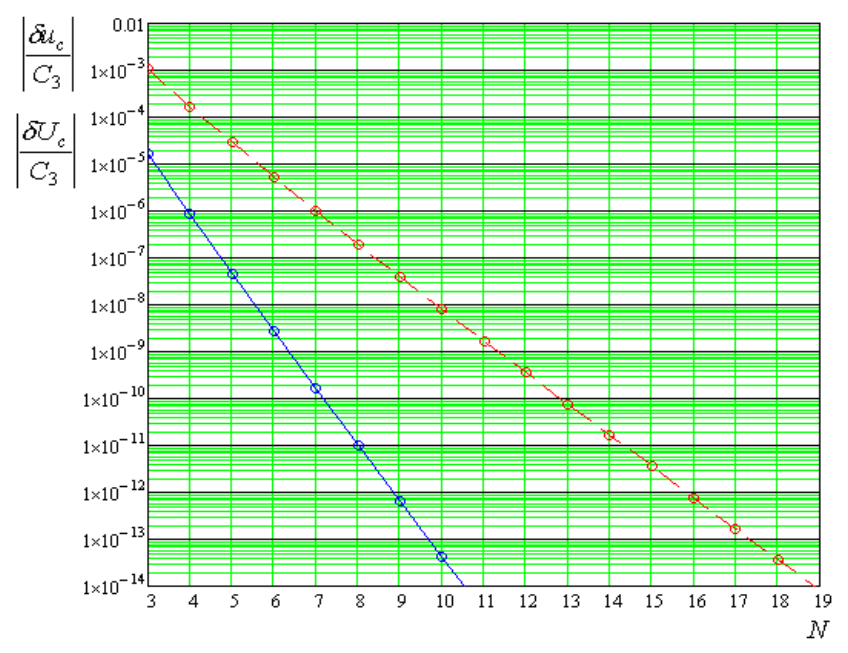

a)

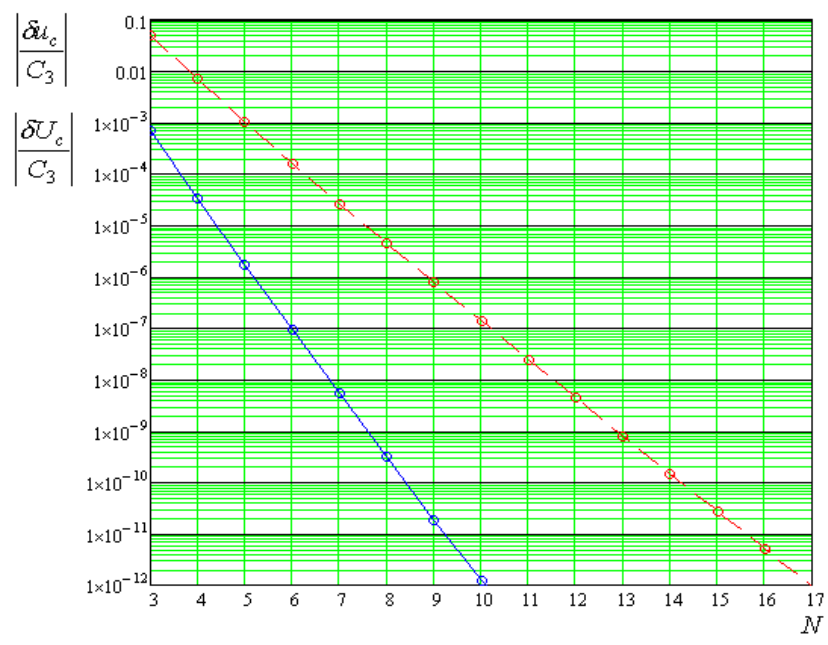

b)

Figure 4. Field description errors at the real antenna array $\left|\frac{\delta u_{c}}{C_{3}}\right|$ (solid lines) and at the virtual antenna array $\left|\frac{\delta U_{c}}{C_{3}}\right|$ (dashed lines) subject to $N$ for: a) $f=25 \mathrm{MHz}, b)-f=100 \mathrm{MHz}$

On Fig. 4 a) you can see dependency of $\left|\frac{\delta u_{c}}{C_{3}}\right|$ and $\left|\frac{\delta U_{c}}{C_{3}}\right|$ on $N$ for $25 \mathrm{MHz}$ frequency. It can be seen, that when the number of harmonics on loop $\gamma$ (auxiliary field emitters) increased approx. by 1.8 , the field description error for loop $\xi$ (virtual antenna array) becomes equal to the field description error on real antenna array loop $L$ (whereas $\left.r_{\xi} / r_{L}=3\right)$.

At $100 \mathrm{MHz}$ frequency (Fig. $4 \mathrm{~b}$ ), the ratio between the required number of harmonics at the virtual antenna loop and the number of harmonics at the real antenna loop with similar field description errors $\left(\left|\frac{\delta U_{c}}{C_{3}}\right|\right.$ and $\left.\left|\frac{\delta u_{c}}{C_{3}}\right|\right)$ remains approx. the same. For field descriptions, $I_{n}(n)$ and $J_{n}(n)$ were approximated with function $\alpha \cdot \exp (-\beta \cdot n)$ with the following parameters: $\alpha_{1}=170$ and $\beta_{1}=2.6 ; \alpha_{2}=170$ and $\beta_{2}=1.55$.

Considering Kotelnikov theorem [10], it can be said that if the number of field loop counts is equal to the total number of considered field harmonics $(2 N+1)$, field description errors will be equal. Therefore, with the given ratio between the virtual and real antenna array radii $r_{\xi} / r_{L}=3$, the number of auxiliary field emitters should be increased by 1.8 (with $r_{\gamma} / r_{L}=10$ ) so that to reduce virtual array loop field extrapolation error to that of the real antenna array loop.

\section{Numerical Experiments}

To evaluate field extrapolation error for loop $\xi$, some numerical experiments were conducted. On Fig. 1 you can see a model of $0.5 \mathrm{~m}$ radius. antenna array carrier; there are 18 antenna elements in use. Such an arrangement allows to study field extrapolation errors with different numbers of antenna array elements: $N=18$ (here signals from all array elements are used); $N=9$ (here signal from just one element is used); $N=6$ (here signals from two elements are used); $N=3$ (here signals from five elements are used).

For boundary problem of flat EM-wave diffraction with vertical polarization (we used Weiland finite integration technique [19] in a space-time domain) onto a mobile antenna array (Fig. 1) at azimuth angle $\varphi=45^{\circ}$ (the azimuth was taken from the car centerline from the rear compartment to the windscreen). The plane, in which antenna array elements phase centers are located (array coordinates were taken as $x_{u}=0 \mathrm{~m} ; y_{u}=0 \mathrm{~m}$ ) was $0.11 \mathrm{~m}$ distant from the carrier body. For that plane, totalized $E_{z}$ was calculated, i.e. field components in the point with the following coordinates: $\left(x_{1}=0.5 \mathrm{~m} ; y_{1}=0.5 \mathrm{~m}\right)$.

The number of auxiliary linear field emitters in use (to describe the field on the plane, Hankel functions of the first kind and zero order $H_{0}^{1}$ were used) was taken the same as the number of the antenna elements: $N=18 ; N=9 ; N=6$; $N=3$. The emitters were set on concentric circles with the center in $\left(x_{u}=0 \mathrm{~m} ; y_{u}=0 \mathrm{~m}\right)$ with 5 meters radius.

On Fig. 5 you can see frequency dependencies for phase $E_{z}$ - the field components in the above point (the solid lines mean dependencies developed in a rigorous solution of the relevant electrodynamic problem by means Weiland finite integration technique: the maximum space network interval was selected as the lowest of the two following values: $\lambda_{\min } / 15 \quad\left(\lambda_{\min }=1.5 \mathrm{~m}\right.$ - the minimum wave length in a free space corresponding to $200 \mathrm{MHz}$ frequency) and $L_{\min } / 20$ (the minimal dimension of carrier body/antenna array component); the dashed lines mean relevant extrapolated phase values defined by means of the virtual antenna array technique).

On Fig. 5 you can see that field approximation accuracy increases if the number of auxiliary emitters $N$ increases 
from 3 to 9 . However, when $N$ increases from 9 to 18 it does not mean that it will improve accuracy at any frequency because matrix conditioning number $\|A\|$ will rise greatly. To improve EM field extrapolation accuracy, special techniques for linear equations systems with ill-conditioned matrices are recommended $[20,21]$.

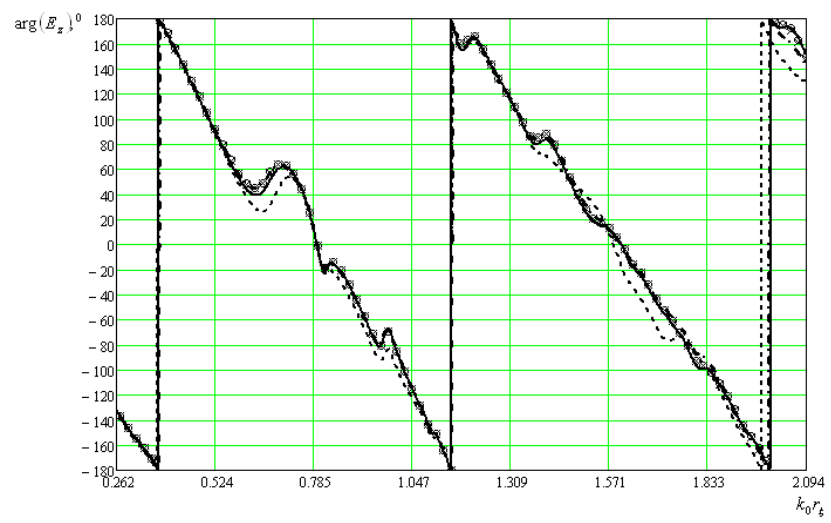

Figure 5. A study of phase $E_{z}$ of field component in point with coordinates ( $x_{1}=0.5 \mathrm{~m} ; y_{1}=0.5$ ) as per 3, 6, 9 and 18 known values measured on a 0.5 m radius circle

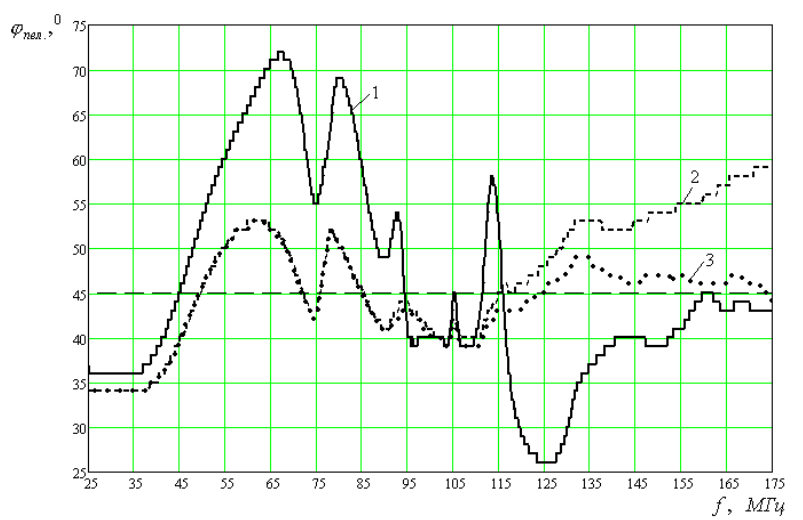

Figure 6. Bearings dependencies with true emitter azimuth value $\varphi_{R F S}=45^{0}: \because$ curve 1 is a real antenna array including 18 elements with $0.5 \mathrm{~m}$ radius; curve 2 is a virtual antenna array with $1.5 \mathrm{~m}$ radius, this array is formed with 9 field counts measured with the real antenna array via just 1 element (element $1,3, \ldots$ 17); curve 3 - is a virtual antenna array comprising 36 elements with $1.5 \mathrm{~m}$ radius, this antenna array is formed with 18 field counts measured with the real antenna array. To plot bearings, interferential correlational phase amplitude technique with reference signals was used; the signals corresponding with wave distribution in a free space

On Fig. 6 you can see bearings frequency dependencies with true emitter azimuth value $\varphi_{R F S}=45^{\circ}$ : curve 1 is a real antenna array including 18 elements with $0.5 \mathrm{~m}$ radius; curve 2 is a virtual antenna array with $1.5 \mathrm{~m}$ radius, this array is formed with 9 field counts measured with the real antenna array via just 1 element (element $1,3, \ldots 17$ ); curve 3 - is a virtual antenna array comprising 36 elements with $1.5 \mathrm{~m}$ radius, this antenna array is formed with 18 field counts measured with the real antenna array. To plot bearings, interferential correlational phase amplitude technique with reference signals was used; the signals corresponding with wave distribution in a free space. On Fig. 6 you can see that if the real antenna array elements number is increased from 9 to 18 , then direction finding accuracy at $125-175 \mathrm{MHz}$ frequency will be also increased greatly. With this, the virtual antenna array technique enables to cut down direction finding error mean square deviation from $11.940^{\circ}$ to $5.030^{\circ}$ within $25-175$ $\mathrm{MHz}$ frequency range.

\section{Nature Experiments}

To study virtual antenna array theory effectiveness with field measurement data, IRCOS Argument direction finder (Fig. 7) was used.

On Fig. 8 you can see bearings frequency dependencies measured with a real antenna array (curve 1) as well as with a $1.2 \mathrm{~m}$ radius virtual antenna array (the " 2 " means a measured bearing dependency plotted by means of direction finding phase technique; the " 3 " means the same but with a phase-amplitude technique).

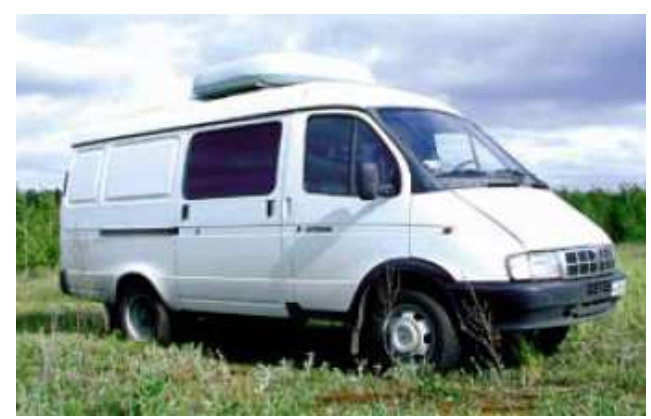

Figure 7. Argument direction finder (7 antenna elements are set on a $0.54 \mathrm{~m}$ radius circle).

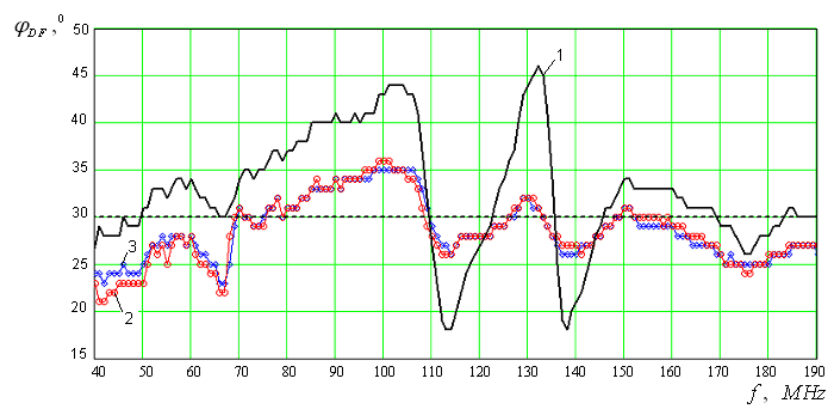

a)

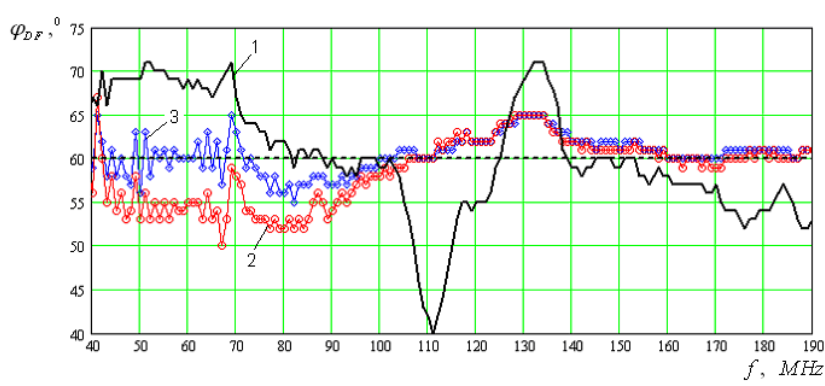

b)

Figure 8. Bearings dependencies plotted using the real antenna array (curve 1) and the virtual antenna array with $12 \mathrm{~m}$ radius (curve 2 - phase technique, curve 3 - phase and amplitude technique). The true value of the emitter azimuth is 30 degrees (a) and 60 degrees (b). 
The above dependencies imply that direction finding errors can be reduced twofold or even fourfold when using virtual antenna array techniques. At the same time, direction finding error RMS can be reduced twofold or even fourfold. The maximum virtual antenna array effect is observed in the frequency range which corresponds to the most intense resonances of the real antenna array carrier body (approx. 90 $150 \mathrm{MHz}$ ). A further increase of the virtual antenna array radius (more than threefold against the real antenna array dimensions) will not further improve direction finding accuracy because there occur errors of phase/amplitude measurement for signals at the real antenna array outputs and they affect field approximation accuracy (in the space around the real antenna array). By means of virtual antenna array technique, it was established that the maximum direction finding accuracy improvement could be achieved when the ration between the virtual and real antenna array radii ranges from 2 to 3 .

\section{Conclusion}

The proposed virtual antenna array technique can be used not only in radio applications like: radio communication, radio location, radio navigation, radio electronic warfare, etc. but in a number of optical and acoustical applications. It can be used for description of electromagnetic or acoustical field near diffusers of random shape, dimensions and material properties. With this, it does not require a priori data about diffusers (antenna array carrier body, mast or other diffusing objects) and their parameters.

In particular, the virtual antenna array technique can be used for improvement of different radio, optical and acoustic system performance: to increase measurement accuracy of radio and acoustical emitters coordinates, improve equipment resolution for angular and linear coordinates, disambiguation of emitters direction finding, improve radio/optical/acoustic equipment throughput, increase accuracy of study of a priori unknown diffusers properties (geometry, materials, etc.) by means of a virtual antenna array with a random number of elements at random positions.

The proposed technique enables to restore the structure of electromagnetic or acoustic field near a random diffuser with a priori unknown parameters. The results, i.e. improvement of radio/optical/acoustic system performance are achieved by means of restoration of the field structure in the space with the minimum distortion of received or emitted waves caused by the diffuser.

Also, it should be noted that the virtual antenna array of random configuration and random number of elements enables to optimize the structure and parameters of the real antenna array so that to minimize its directional pattern distortion by the carrier's body (support mast with guide ropes, equipment and other nearest diffusers). Besides, it does not require a great number of calculations or field experiments or studies.

To restore the structure of electromagnetic or acoustical field, a number of algorithms can be used. E.g.: algorithms based on harmonic functions (scalar and vector fields) properties, complex variable theory (in particular, analytical functions theory and applications), field approximation inside and outside of the stationary/mobile or onboard antenna array with a system of functions in the spatial domain in question: $(V, S)$.

\section{References}

[1] B. Friedlander. Direction finding with an interpolated array. // Proc. IEEE Int. Conf. Acoust., Speech, Signal Processing, Apr. 1990.

[2] Tuncer E., Friedlander B. Classical and Modern Direction-of-Arrival Estimation. USA: AP. 2009. 429 P.

[3] M. Pesavento, A. B. Gershman, and Zhi-Quan Luo. Robust array interpolation using second-order cone programming. IEEE Signal Processing Letters, vol. 9 no. 1, pp. 8-11, Jan. 2002.

[4] M. Buhren, M. Pesavento, and J. F. Bohme. A new approach to array interpolation by generation of artificial shift invariances: interpolated ESPRIT. Proceedings IEEE Int. Conf. Acoustics, Speech, and Signal Proces. (ICASSP), vol. 5, pp. 205-208, 2003.

[5] Hyberg P. Antenna Array Mapping for DOA Estimation in Radio Signal Reconnaissance. PhD thesis. - Royal Institute of Technology (KTH), Stockholm, Sweden. - 2005.

[6] F. Belloni, A. Richter, and V. Koivunen. Reducing Excess Variance in Beamspace Methods for Uniform Circular Array. In Proceedings of the IEEE Workshop on Statistical Signal Processing (SSP), Bordeaux, France, July 17-20, 2005.

[7] S. Chen, B. Mulgrew, and P. M. Grant, "A clustering technique for digital communications channel equalization using radial basis function networks," IEEE Trans. Neural Networks, vol. 4, pp. 570-578, Jul. 1993.

[8] Belloni F., Richter A., Koivunen V. DOA Estimation via Manifold Separation For Arbitrary Array Structures // IEEE Trans. Signal Processing, 2007, vol. 55, № 10, pp. 4800-4810.

[9] Advances in Direction-of-Arrival Estimation / S. Chandran. Norwood: ARTECH HOUSE. 2006. 474 P.

[10] Antenna Array Including Virtual Antenna. P. van Rooyen, P. Roux. Patent US 7,605,755B2, 20.10.2009 (prior publication data US 2008/0303719 A1, 11.12.2008).

[11] (in Russian) Transl.: V.I. Glazyev, R.A. Zatserkovsky, O.V. Smidovich, "The method of phantoms in the theory of antenna arrays," Materials of Acoustic Symposium "Consonans-2003". Kiev. 2003. Publisher NAN of Ukraine. PP. 67-72.

[12] (in Russian) Transl.: Yu.G. Pasternak, Yu.A. Rembovsky, "Field structure in the region of the mobile circular antenna array," Antennas. 2007. № 1 (116). PP. 30-34.

[13] (in Russian) Transl.: Yu.G. Pasternak, Yu.A. Rembovsky, "The method of recovery of the electromagnetic field on plane near three-dimensional scatterer," Antennas. 2007. № 7 (122). PP. 43-48.

[14] A.V. Ashikhmin, Yu.G. Pasternak, Yu.A. Rembovsky, "The method of synthesis of a "virtual" array and researching of the possibility of using it to improve the performance of mobile and stationary radio direction finders," (in Russian) Transl.: Antennas. 2008. № 10 (137). PP. 34-46. 
[15] (in Russian) Transl.: A.V. Ashikhmin, S.V. Korochin, Yu.G. Pasternak, Yu.A. Rembovsky, "The method of synthesis of direction finding antenna array on the case of the mobile carrier" Radiolocation and communication. 2009. № 8. PP. 18-23.

[16] (in Russian) Transl.: A.S. Ilyinsky, V.V. Kravtsov, A.G. Sveshnikov, "Mathematical models of electrodynamics," Moscow: Higher School. 1991. 224 p.

[17] (in Russian) Transl.: M.A. Aleksidze, "The fundamental functions in approximate solutions of boundary problems," Moscow: Nauka, 1991. 352 p.

[18] (in Russian) Transl.: G. Korn, T. Korn, "Mathematical
Handbook for Scientists and Engineers," Moscow: Nauka, 1973. $832 \mathrm{p}$.

[19] Weiland T. A discretization method for the solution of Maxwell`s equations for six-component fields // Electronics and Communication, 1977. V. 31. PP. 116-120.

[20] (in Russian) Transl.: J.A. Bakhvalov, S.Yu. Knyazev, A.A. Shcherbakov, "Mathematical modeling of physical fields by point sources method," Izvestiya RAN. A physical series. 2008. T. 72, № 9. PP. 1259-1261.

[21] (in Russian) Transl.: N.N. Kalitkin, "Numerical methods," Nauka. 1978. 512 p. 